\title{
THERMOELASTIC STABILITY OF THICK IMPERFECT FUNCTIONALLY GRADED PLATES
}

\author{
Hoang Van Tung ${ }^{1}$, Nguyen Dinh Duc ${ }^{2}$ \\ ${ }^{1}$ Hanoi Architectural University, Vietnam \\ ${ }^{2}$ Vietnam National University, Hanoi
}

\begin{abstract}
This paper investigates buckling of thick functionally graded plates with initial geometrical imperfection under thermal loadings. The equilibrium, stability, and compatibility equations of an imperfect functionally graded plate are derived using the third order shear deformation theory. Material properties are assumed to be temperatureindependent and graded in the thickness direction according to a simple power law distribution in terms of the thickness coordinate variable. By Galerkin method, the resulting equations are solved to obtain closed-form solutions of critical buckling temperature difference. Two types of thermal loading, uniform temperature rise and nonlinear temperature change across the thickness are considered. Buckling analysis for a simply supported rectangular imperfect functionally graded plate shows effects of geometry and material parameters, shear deformation and imperfection on critical buckling temperature.
\end{abstract}

\section{INTRODUCTION}

By high performance heat resistant capacity, Functionally Graded Materials (FGMs) have received much attention for structural applications in ultrahigh temperature environments and extremely large temperature gradient such as aircraft, space vehicles, nuclear plants, and many other applications. By varying smoothly and continously of mechanical properties from one surface to the other, FGMs eliminates interface problems and stress concentrations. Javaheri and Eslami reported mechanical and thermal buckling of rectangular functionally graded plates by using the classical theory $[2,3]$ and the third order shear deformation theory $[4,5]$. They used energy method to derive governing equations that are analytically solved to obtain the closed-form solutions of critical loading. Lanhe [6] used first order shear deformation theory to derive closed-form relations for buckling temperature difference of simply supported moderately thick rectangular FGM plates. Three-dimensional thermal buckling analysis of functionally graded composite plates, using finite element method, is reported by $\mathrm{Na}$ and Kim [7]. The research on thermoelastic stability of FGM cylindrical shells is introduced by Eslami and his associates $[9,10]$ and Lanhe et al. [11] according to analytical approach.

The initial geometrical imperfections are unavoidable and inherent in many real structures. Therefore, many investigations are conducted on the stability of imperfect structures such as works reported by Eslami and his co-workers [12-15], Murphy and Ferreira [16] for 
isotropic and orthotropic imperfect structures under mechanical and thermal loads. Recently, Shariat and Eslami have extended their preceding works to investigate the stability of FGM plates with initial imperfection by using classical [18] and first order [19] theories. Yang et al. [20] have suggested a general form of imperfection function including local and global imperfection modes and analyzed their sensitivity on postbuckling behavior of higher order shear deformable FGM plates.

The present paper, mainly motivated by foregoing works $[18,19]$, investigates thermal buckling of thick rectangular FGM plates with initial geometrical imperfections. Formulation is based on the Reddy's third order shear deformation theory. By Galerkin method, the closed-form solutions of critical buckling temperature differences are derived. Buckling analysis for a simply supported FGM plate subjected to two types of thermal loadings shows effects of plate geometry and material parameters, shear deformation and imperfection on the critical buckling temperature.

\section{FUNCTIONALLY GRADED PLATES}

Consider a rectangular functionally graded plate of length $a$, width $b$, and thickness $h$, referred to the rectangular Cartesian coordinates $(x, y, z)$, where $(x, y)$ plane coincides with middle surface of the plate and $z$ is the thickness coordinate $(-h / 2 \leqslant z \leqslant h / 2)$.

By applying a simple power law distribution, the volume fractions of metal and ceramic, $V_{m}$ and $V_{c}$, are obtained as follows $[3,4,6,8,18,19]$

$$
V_{c}(z)=\left(\frac{2 z+h}{2 h}\right)^{k}, \quad V_{m}(z)=1-V_{c}(z)
$$

where volume fraction index $k$ is a nonnegative number that defines the material distribution and can be chosen to optimize the structural respone.

We assume that the effective properties $P_{\text {eff }}$ of functionally graded plate, such as the modulus of elasticity $E$, the coefficient of thermal expansion $\alpha$, and the coefficient of thermal conduction $K$, change in the thickness direction $z$ and can be determined by the linear rule of mixture as $[3,4,5,6]$

$$
P_{\text {eff }}=P_{c} V_{c}+P_{m} V_{m}
$$

where $P$ denotes a temperature-independent material property, and subscripts $m$ and $c$ stand for the metal and ceramic constituents, respectively. From Eqs. (1), (2), the effective properties of FGM plate can be written as follows, while Poisson's ratio $\nu$ is assumed to be constant for simplicity.

$$
[E(z), \alpha(z), K(z)]=\left[E_{m}, \alpha_{m}, K_{m}\right]+\left[E_{c m}, \alpha_{c m}, K_{c m}\right]\left(\frac{2 z+h}{2 h}\right)^{k}, \nu(z)=\nu
$$

where

$$
E_{c m}=E_{c}-E_{m} \quad, \alpha_{c m}=\alpha_{c}-\alpha_{m} \quad, K_{c m}=K_{c}-K_{m} .
$$

\section{GOVERNING EQUATIONS}

In the present study, the Reddy's third order shear deformation theory (TSDT) [21] is used to obtain the equilibrium, stability, and compatibility equations as well as critical 
buckling loading relations. The strains across the plate thickness at a distance $z$ from the mid-plane are $[4,5]$

$$
\begin{gathered}
\left(\begin{array}{c}
\varepsilon_{x} \\
\varepsilon_{y} \\
\gamma_{x y}
\end{array}\right)=\left(\begin{array}{c}
\varepsilon_{x}^{0} \\
\varepsilon_{y}^{0} \\
\gamma_{x y}^{0}
\end{array}\right)+z\left(\begin{array}{c}
k_{x}^{0} \\
k_{y}^{0} \\
k_{x y}^{0}
\end{array}\right)+z^{3}\left(\begin{array}{c}
k_{x}^{2} \\
k_{y}^{2} \\
k_{x y}^{2}
\end{array}\right) \\
\left(\begin{array}{c}
\gamma_{x z} \\
\gamma_{y z}
\end{array}\right)=\left(\begin{array}{c}
\gamma_{x z}^{0} \\
\gamma_{y z}^{0}
\end{array}\right)+z^{2}\left(\begin{array}{c}
k_{x z}^{1} \\
k_{y z}^{1}
\end{array}\right)
\end{gathered}
$$

where

$$
\begin{aligned}
& \left(\begin{array}{c}
\varepsilon_{x}^{0} \\
\varepsilon_{y}^{0} \\
\gamma_{x y}^{0}
\end{array}\right)=\left(\begin{array}{c}
u_{, x}+w_{, x}^{2} / 2 \\
v_{, y}+w_{, y}^{2} / 2 \\
u_{, y}+v_{, x}+w_{, x} w_{, y}
\end{array}\right),\left(\begin{array}{c}
k_{x}^{0} \\
k_{y}^{0} \\
k_{x y}^{0}
\end{array}\right)=\left(\begin{array}{c}
\phi_{x, x} \\
\phi_{y, y} \\
\phi_{x, y}+\phi_{y, x}
\end{array}\right), \\
& \left(\begin{array}{c}
k_{x}^{2} \\
k_{y}^{2} \\
k_{x y}^{2}
\end{array}\right)=-c_{1}\left(\begin{array}{c}
\phi_{x, x}+w_{, x x} \\
\phi_{y, y}+w_{, y y} \\
\phi_{x, y}+\phi_{y, x}+2 w_{, x y}
\end{array}\right), \\
& \left(\begin{array}{c}
\gamma_{x z}^{0} \\
\gamma_{y z}^{0}
\end{array}\right)=\left(\begin{array}{c}
\phi_{x}+w_{, x} \\
\phi_{y}+w_{, y}
\end{array}\right),\left(\begin{array}{c}
k_{x z}^{1} \\
k_{y z}^{1}
\end{array}\right)=-3 c_{1}\left(\begin{array}{c}
\phi_{x}+w_{, x} \\
\phi_{y}+w_{, y}
\end{array}\right)
\end{aligned}
$$

in which $c_{1}=4 / 3 h^{2}, \varepsilon_{x}, \varepsilon_{y}$ are the normal strains, $\gamma_{x y}$ is the shear strain at the middle surface of the plate, and $\gamma_{x z}, \gamma_{y z}$ are the transverse shear deformations, also, $(u, v, w)$ are the mid-plane displacement components, and $\phi_{x}, \phi_{y}$ are the slope rotations in the $(x, z)$ and $(y, z)$ planes, respectively.

Hooke's law for a plate is defined as

$$
\begin{gathered}
\sigma_{x}=\frac{E}{1-\nu^{2}}\left[\varepsilon_{x}+\nu \varepsilon_{y}-(1+\nu) \alpha \Delta T\right] \\
\sigma_{y}=\frac{E}{1-\nu^{2}}\left[\varepsilon_{y}+\nu \varepsilon_{x}-(1+\nu) \alpha \Delta T\right] \\
{\left[\sigma_{x y}, \sigma_{x z}, \sigma_{y z}\right]=\frac{E}{2(1+\nu)}\left[\gamma_{x y}, \gamma_{x z}, \gamma_{y z}\right]}
\end{gathered}
$$

The force and moment resultants of the plate are defined as

$$
\begin{aligned}
\left(N_{i}, M_{i}, P_{i}\right) & =\int_{-h / 2}^{h / 2} \sigma_{i}\left(1, z, z^{3}\right) d z \quad i=x, y, x y \\
\left(Q_{i}, R_{i}\right) & =\int_{-h / 2}^{h / 2} \sigma_{j}\left(1, z^{2}\right) d z \quad i=x, y ; j=x z, y z .
\end{aligned}
$$


Substituting Eqs. (3), (5), (6) and (8) into Eqs. (9) gives the constitutive relations as [4, 5]

$$
\begin{aligned}
\left(N_{x}, M_{x}, P_{x}\right)= & \frac{1}{1-\nu^{2}}\left[\left(E_{1}, E_{2}, E_{4}\right)\left(\varepsilon_{x}^{0}+\nu \varepsilon_{y}^{0}\right)+\left(E_{2}, E_{3}, E_{5}\right)\left(k_{x}^{0}+\right.\right. \\
& \left.\left.\nu k_{y}^{0}\right)+\left(E_{4}, E_{5}, E_{7}\right)\left(k_{x}^{2}+\nu k_{y}^{2}\right)-(1+\nu)\left(\Phi_{1}, \Phi_{2}, \Phi_{4}\right)\right] \\
\left(N_{y}, M_{y}, P_{y}\right)= & \frac{1}{1-\nu^{2}}\left[\left(E_{1}, E_{2}, E_{4}\right)\left(\varepsilon_{y}^{0}+\nu \varepsilon_{x}^{0}\right)+\left(E_{2}, E_{3}, E_{5}\right)\left(k_{y}^{0}+\right.\right. \\
& \left.\left.\nu k_{x}^{0}\right)+\left(E_{4}, E_{5}, E_{7}\right)\left(k_{y}^{2}+\nu k_{x}^{2}\right)-(1+\nu)\left(\Phi_{1}, \Phi_{2}, \Phi_{4}\right)\right] \\
\left(N_{x y}, M_{x y}, P_{x y}\right)= & \frac{1}{2(1+\nu)}\left[\left(E_{1}, E_{2}, E_{4}\right) \gamma_{x y}^{0}+\left(E_{2}, E_{3}, E_{5}\right) k_{x y}^{0}+\left(E_{4}, E_{5}, E_{7}\right) k_{x y}^{2}\right] \\
\left(Q_{x}, R_{x}\right)= & \frac{1}{2(1+\nu)}\left[\left(E_{1}, E_{3}\right) \gamma_{x z}^{0}+\left(E_{3}, E_{5}\right) k_{x z}^{1}\right] \\
\left(Q_{y}, R_{y}\right)= & \frac{1}{2(1+\nu)}\left[\left(E_{1}, E_{3}\right) \gamma_{y z}^{0}+\left(E_{3}, E_{5}\right) k_{y z}^{1}\right]
\end{aligned}
$$

where

$$
\begin{aligned}
\left(E_{1}, E_{2}, E_{3}, E_{4}, E_{5}, E_{7}\right) & =\int_{-h / 2}^{h / 2}\left(1, z, z^{2}, z^{3}, z^{4}, z^{6}\right) E(z) d z \\
\left(\Phi_{1}, \Phi_{2}, \Phi_{4}\right) & =\int_{-h / 2}^{h / 2}\left(1, z, z^{2}\right) E(z) \alpha(z) \Delta T(x, y, z) d z
\end{aligned}
$$

Substituting Eqs. (3) into Eqs. (11) gives coefficients $E_{i},(i=1-7)$ (see Appendix).

The nonlinear equilibrium equations of a perfect functionally graded plate based on the third order shear deformation theory are $[4,5,20]$

$$
\begin{aligned}
& N_{x, x}+N_{x y, y}=0, N_{x y, x}+N_{y, y}=0, \\
& Q_{x, x}+Q_{y, y}-3 c_{1}\left(R_{x, x}+R_{y, y}\right)+c_{1}\left(P_{x, x x}+2 P_{x y, x y}+P_{y, y y}\right) \\
& +N_{x} w_{, x x}+2 N_{x y} w_{, x y}+N_{y} w_{, y y}=0 \\
& M_{x, x}+M_{x y, y}-Q_{x}+3 c_{1} R_{x}-c_{1}\left(P_{x, x}+P_{x y, y}\right)=0 \\
& M_{x y, x}+M_{y, y}-Q_{y}+3 c_{1} R_{y}-c_{1}\left(P_{x y, x}+P_{y, y}\right)=0
\end{aligned}
$$

Assuming that the temperature varies with respect to the thickness direction only and eliminating the variables $u, v, \phi_{x}, \phi_{y}$ from the last three equations, Eqs. (12) may be reduced to a set of three equations as

$$
\begin{aligned}
& N_{x, x}+N_{x y, y}=0, N_{x y, x}+N_{y, y}=0 \\
& c_{1}^{2}(E B / D-C) \Delta^{3} w+\left(c_{1} B / D+1\right) F \Delta^{2} w+\left(1-c_{1} E / D\right) \Delta\left(N_{x} w_{, x x}\right. \\
& \left.\quad+2 N_{x y} w_{, x y}+N_{y} w_{, y y}\right)-F / D\left(N_{x} w_{, x x}+2 N_{x y} w_{, x y}+N_{y} w_{, y y}\right)=0
\end{aligned}
$$

where $\Delta=\partial^{2} / \partial x^{2}+\partial^{2} / \partial y^{2}$, and

$$
\begin{aligned}
& A=\frac{E_{1} E_{3}-E_{2}^{2}}{\left(1-\nu^{2}\right) E_{1}}, B=\frac{E_{1} E_{5}-E_{2} E_{4}}{\left(1-\nu^{2}\right) E_{1}}, C=\frac{E_{1} E_{7}-E_{4}^{2}}{\left(1-\nu^{2}\right) E_{1}} \\
& D=A-c_{1} B, E=B-c_{1} C, F=\frac{1}{2(1+\nu)}\left(E_{1}-6 c_{1} E_{3}+9 c_{1}^{2} E_{5}\right) .
\end{aligned}
$$


For a slightly imperfect plate, let $w^{*}(x, y)$ denotes a known small imperfection. This parameter represents a small deviation of the plate middle plane from a flat shape. When imperfection is considered, equilibrium Eqs. (13) are modified as (see [18]).

$$
\begin{aligned}
& N_{x, x}+N_{x y, y}=0, N_{x y, x}+N_{y, y}=0 \\
& c_{1}^{2}(E B / D-C) \Delta^{3} w+\left(c_{1} B / D+1\right) F \Delta^{2} w+\left(1-c_{1} E / D\right) \Delta\left[N_{x}(\right. \\
& \left.\left.w_{, x x}+w_{, x x}^{*}\right)+2 N_{x y}\left(w_{, x y}+w_{, x y}^{*}\right)+N_{y}\left(w_{, y y}+w_{, y y}^{*}\right)\right] \\
& -\frac{F}{D}\left[N_{x}\left(w_{, x x}+w_{, x x}^{*}\right)+2 N_{x y}\left(w_{, x y}+w_{, x y}^{*}\right)+N_{y}\left(w_{, y y}+w_{, y y}^{*}\right)\right]=0 .
\end{aligned}
$$

When only the first order shear deformation is taken into consideration, Eqs. (15) are reduced to those reported by Shariat and Eslami [19]. Also, when the shear deformation is ignored, Eqs. (15) are identical to results obtained by Shariat and Eslami [18].

The stability equation of the imperfect FGM plate may be derived by the adjacent equilibrium criterion [1] as follows

$$
\begin{aligned}
& N_{x 1, x}+N_{x y 1, y}=0, N_{x y 1, x}+N_{y 1, y}=0 \\
& c_{1}^{2}(E B / D-C) \Delta^{3} w_{1}+\left(c_{1} B / D+1\right) F \Delta^{2} w_{1}+\left[\left(1-c_{1} E / D\right) \Delta\right. \\
& \quad-F / D]\left(N_{x 0} w_{1, x x}+2 N_{x y 0} w_{1, x y}+N_{y 0} w_{1, y y}\right)+\left[\left(1-c_{1} E / D\right) \Delta\right. \\
& \quad-F / D]\left[N_{x 1}\left(w_{0, x x}+w_{, x x}^{*}\right)+2 N_{x y 1}\left(w_{0, x y}+w_{, x y}^{*}\right)+N_{y 1}\left(w_{0, y y}+w_{, y y}^{*}\right)\right]=0
\end{aligned}
$$

where subscript 1 refers to the stability state and the subscript 0 refers to the state of equilibrium conditions. Also, $N_{x 1}, N_{y 1}$, and $N_{x y 1}$ represent the parts of increments of force resultant that are linear in $u_{1}, v_{1}$ and $w_{1}$, whereas $N_{x 0}, N_{y 0}$, and $N_{x y 0}$ are prebuckling force resultants. Considering the first two of Eqs. (16), a stress function $f$ may be defined as

$$
N_{x 1}=f_{, y y}, N_{y 1}=f_{, x x}, N_{x y 1}=-f_{, x y}
$$

Substituting Eqs. (17) into Eqs. (16) gives

$$
\begin{aligned}
& c_{1}^{2}(E B / D-C) \Delta^{3} w_{1}+\left(c_{1} B / D+1\right) F \Delta^{2} w_{1}+\left[\left(1-c_{1} E / D\right) \Delta\right. \\
& \quad-F / D]\left(N_{x 0} w_{1, x x}+2 N_{x y 0} w_{1, x y}+N_{y 0} w_{1, y y}\right)+\left[\left(1-c_{1} E / D\right) \Delta\right. \\
& \quad-F / D]\left[f_{, y y}\left(w_{0, x x}+w_{, x x}^{*}\right)-2 f_{, x y}\left(w_{0, x y}+w_{, x y}^{*}\right)+f_{, x x}\left(w_{0, y y}+w_{, y y}^{*}\right)\right]=0 .
\end{aligned}
$$

The above stability equation includes two dependent unknowns, $w_{1}$ and $f$. To obtain a second equation relating these two unknowns, the compatibility equation may be used. The membrance strain components $\varepsilon_{x 1}^{0}, \varepsilon_{y 1}^{0}, \gamma_{x y 1}^{0}$ which are linear in $u_{1}, v_{1}, w_{1}$ may be written from Eqs. (7) with considering imperfection as follows

$$
\begin{aligned}
\varepsilon_{x 1}^{0} & =u_{1, x}+\left(w_{0, x}+w_{, x}^{*}\right) w_{1, x}, \varepsilon_{y 1}^{0}=v_{1, y}+\left(w_{0, y}+w_{, y}^{*}\right) w_{1, y}, \\
\gamma_{x y 1}^{0} & =u_{1, y}+v_{1, x}+\left(w_{0, x}+w_{, x}^{*}\right) w_{1, y}+\left(w_{0, y}+w_{, y}^{*}\right) w_{1, x} .
\end{aligned}
$$

Using Eqs. (19), the geometrical compatibility equation is written as

$$
\varepsilon_{x 1, y y}^{0}+\varepsilon_{y 1, x x}^{0}-\gamma_{x y 1, x y}^{0}=2\left(w_{0, x y}+w_{, x y}^{*}\right) w_{1, x y}-\left(w_{0, x x}+w_{, x x}^{*}\right) w_{1, y y}-\left(w_{0, y y}+w_{, y y}^{*}\right) w_{1, x x} .
$$


The membrance strains $\varepsilon_{i j}^{0}$ expressed in terms of force resultants due to Eqs. (10), then the results are substituted in Eq. (20) and with the aid of Eqs. (17) leads to compatibility equation of an imperfect FGM plate as

$$
\frac{1}{E_{1}} \Delta^{2} f-2 w_{1, x y}\left(w_{0, x y}+w_{, x y}^{*}\right)+w_{1, x x}\left(w_{0, y y}+w_{, y y}^{*}\right)+w_{1, y y}\left(w_{0, x x}+w_{, x x}^{*}\right)=0 .
$$

Eqs. (15), (18) and (21) are the basic equations used to obtain the critical buckling load of an imperfect plate.

\section{BUCKLING ANALYSIS}

In this section, the closed-form relations of buckling loading of imperfect FGM rectangular plate under two types of thermal loadings are presented. The plate is assumed simply supported in bending and rigidly fixed in extension.

\subsection{Uniform temperature rise}

Environment temperature is assumed to be raised uniformly from initial value $T_{i}$ to final one $T_{f}$ and temperature difference $\Delta T=T_{f}-T_{i}$ is considered as a constant. Solving the membrance form of equilibrium equations, gives the prebuckling force resultants $[3,4$, $6,18,19]$ as

$$
N_{x 0}=-\frac{\Phi_{1}}{1-\nu}, \quad N_{y 0}=-\frac{\Phi_{1}}{1-\nu}, \quad N_{x y 0}=0 .
$$

The simply supported boundary conditions are defined as

$$
\begin{gathered}
u=v=w=\phi_{y}=P_{x}=M_{x}=0 \\
u=v=w=\phi_{x}=P_{y}=M_{y}=0
\end{gathered}
$$

Considering the boundary conditions, the imperfections of the plate are assumed as $[18$, $19]$

$$
w^{*}=\mu h \sin \frac{m \pi x}{a} \sin \frac{n \pi y}{b}, m, n=1,2, \ldots
$$

where the coefficient $\mu$ varies between 0 and 1 and $\mu h$ represents the imperfection size. Also, $m$ and $n$ are number of haft waves in $x$ and $y$ directions, respectively.

The following approximate solution is seen to satisfy both the equilibrium equation (15) and the boundary conditions (23)

$$
w_{0}=W_{m n} \sin \frac{m \pi x}{a} \sin \frac{n \pi y}{b}
$$

where $W_{m n}$ is a constant coefficient. Substituting Eqs. (22), (24), (25) into the third equilibrium equation (15), the constant $W_{m n}$ is obtained as

$$
W_{m n}=\frac{\left[\left(1-c_{1} \frac{E}{D}\right) L_{m n}+\frac{F}{D}\right] \frac{\Phi_{1}}{1-\nu} \mu h}{-c_{1}^{2}\left(\frac{E B}{D}-C\right) L_{m n}^{2}+\left[F\left(c_{1} \frac{B}{D}+1\right)-\frac{\Phi_{1}}{1-\nu}\left(1-c_{1} \frac{E}{D}\right)\right] L_{m n}-\frac{\Phi_{1}}{1-\nu} \frac{F}{D}}
$$

where

$$
L_{m n}=(m \pi / a)^{2}+(n \pi / b)^{2} .
$$


Substituting Eqs. (22), (25), (26) and Eq. (24) into Eqs. (18) and (21) results in two equations with two incremental variables $w_{1}$ and $f$, and with the consideration of the boundary conditions (23), we assume the following approximate solutions

$$
w_{1}=E_{m n} \sin \frac{m \pi x}{a} \sin \frac{n \pi y}{b} ; \quad f=F_{m n} \sin \frac{m \pi x}{a} \sin \frac{n \pi y}{b}
$$

where $E_{m n}$ and $F_{m n}$ are constant coefficients. Substituting the approximate solutions (28) into Eqs. (18) and (21) and applying Galerkin procedure to system of resulting equations yield the thermal parameter $\Phi_{1}$ from condition for nontrivial solutions. After that, the value of buckling temperature difference $\Delta T$ is obtained using Eqs. (11) as

$$
\Delta T=\frac{(1-\nu) \pi^{2}}{P B_{h}^{2}} \frac{\bar{F}\left(\frac{4 \bar{B}}{3 \bar{D}}+1\right) B_{h}^{2} R_{m n}-\frac{16}{9}\left(\frac{\overline{E B}}{\bar{D}}-\bar{C}\right) \pi^{2} R_{m n}^{2}+\pi^{2}\left(\bar{I}_{m n}\right)^{1 / 3}}{\frac{\bar{F}}{\bar{D}} B_{h}^{2}+\left(1-\frac{4 \bar{E}}{3 \bar{D}}\right) \pi^{2} R_{m n}}
$$

where

$$
\begin{gathered}
\bar{I}_{m n}=\frac{1024 \bar{E}_{1} m^{2} n^{2} \mu^{2} B_{a}^{4}}{9 \pi^{10} R_{m n}}\left[\bar{F}\left(\frac{4 \bar{B}}{3 \bar{D}}+1\right) B_{h}^{2}-\frac{16 \pi^{2}}{9}\left(\frac{\overline{E B}}{\bar{D}}-\bar{C}\right) R_{m n}\right]^{2} \\
\quad\left[2\left(1-\frac{4 \bar{E}}{3 \bar{D}}\right) \pi^{2} R_{m n}+\frac{\bar{F}}{\bar{D}} B_{h}^{2}\right] \\
R_{m n}=B_{a}^{2} m^{2}+n^{2}, B_{a}=b / a, B_{h}=b / h, \bar{E}_{1}=E_{1} / h, \bar{A}=A / h^{3} \\
\bar{B}=B / h^{5}, \bar{C}=C / h^{7}, \bar{D}=D / h^{3}, \bar{E}=E / h^{5}, \bar{F}=F / h \\
P=E_{m} \alpha_{m}+\left(E_{m} \alpha_{c m}+E_{c m} \alpha_{m}\right) /(k+1)+E_{c m} \alpha_{c m} /(2 k+1) .
\end{gathered}
$$

The critical buckling temperature difference $\Delta T_{c r}$ is obtained for the values of $m$ and $n$ that make the preceding expression $\Delta T$ a minimum. When minimization methods are used, critical temperature difference is obtained for $m=n=1$.

$$
\Delta T_{c r}=\frac{(1-\nu) \pi^{2}}{P B_{h}^{2}} \frac{\bar{F}\left(\frac{4 \bar{B}}{3 \bar{D}}+1\right) B_{h}^{2} R_{11}-\frac{16}{9}\left(\frac{\overline{E B}}{\bar{D}}-\bar{C}\right) \pi^{2} R_{11}^{2}+\pi^{2}\left(\bar{I}_{11}\right)^{1 / 3}}{\frac{\bar{F}}{\bar{D}} B_{h}^{2}+\left(1-\frac{4 \bar{E}}{3 \bar{D}}\right) \pi^{2} R_{11}}
$$

Remarks Equation (33) is used to determine the critical temperature difference of imperfect FGM plates under uniform temperature rise. This equation indicates that the critical buckling temperature difference of an imperfect FGM plate is increased in comparison with a perfect one by an imperfection term $\left(\bar{I}_{11}\right)^{1 / 3}$, which directly depends on the imperfection size $\mu$, material and geometrical properties. Specializing this equation gives some limiting cases, as follows.

1. Setting $\mu=0$, Eq. (33) is reduced to equation

$$
\Delta T_{c r}=\frac{(1-\nu) \pi^{2}\left(B_{a}^{2}+1\right)}{P B_{h}^{2}} \frac{\bar{F}\left(\bar{D}+\frac{4}{3} \bar{B}\right) B_{h}^{2}-\frac{16}{9}(\overline{E B}-\overline{C D}) \pi^{2}\left(B_{a}^{2}+1\right)}{\bar{F} B_{h}^{2}+\left(\bar{D}-\frac{4}{3} \bar{E}\right) \pi^{2}\left(B_{a}^{2}+1\right)} .
$$

This equation is used to determine the critical temperature change of perfect FGM plates basing the third order shear deformation theory (TSDT). In case of the first order shear 
deformation theory (FSDT), Eq. (34) is reduced to equation

$$
\Delta T_{c r}^{1}=\frac{(1-\nu) \pi^{2}}{P} \frac{\left(\bar{E}_{1} \bar{E}_{3}-\bar{E}_{2}^{2}\right) \bar{E}_{1}\left(B_{a}^{2}+1\right)}{\bar{E}_{1}^{2} B_{h}^{2}\left(1-\nu^{2}\right)+2(1+\nu)\left(\bar{E}_{1} \bar{E}_{3}-\bar{E}_{2}^{2}\right) \pi^{2}\left(B_{a}^{2}+1\right)}
$$

where

$$
\bar{E}_{1}=E_{1} / h, \bar{E}_{2}=E_{2} / h^{2}, \bar{E}_{3}=E_{3} / h^{3} .
$$

Eq. (35) has been derived by Lanhe [6]. Also, when shear deformation is ignored, referred to as case of the classical plate theory (CPT), Eq. (34) is reduced to result

$$
\Delta T_{c r}^{0}=\frac{1}{P} \frac{\pi^{2}\left(B_{a}^{2}+1\right)\left(\bar{E}_{1} \bar{E}_{3}-\bar{E}_{2}^{2}\right)}{(1+\nu) \bar{E}_{1} B_{h}^{2}}
$$

that obtained by Javaheri and Eslami [3].

2. When only the first order shear deformation is considered, Eq. (33) is derived to equation

$$
\Delta T_{c r 1}=\frac{(1-\nu) \pi^{2}}{P} \frac{\left[B_{a}^{2}+1+\left(\bar{I}_{11}^{1}\right)^{1 / 3}\right]\left(\bar{E}_{1} \bar{E}_{3}-\bar{E}_{2}^{2}\right) \bar{E}_{1}}{\bar{E}_{1}^{2} B_{h}^{2}\left(1-\nu^{2}\right)+2(1+\nu)\left(\bar{E}_{1} \bar{E}_{3}-\bar{E}_{2}^{2}\right) \pi^{2}\left(B_{a}^{2}+1\right)}
$$

where

$$
\bar{I}_{m n}^{1}=\frac{1024 \bar{E}_{1} m^{2} n^{2} \mu^{2} B_{a}^{4}}{9 \bar{A} \pi^{4} R_{m n}}\left(1+\frac{4(1+\nu) \bar{A} \pi^{2}}{\bar{E}_{1} B_{h}^{2}} R_{m n}\right) .
$$

Eq. (38) has been obtained by Shariat and Eslami [19]. Clearly, for perfect FGM plates $(\mu=0)$, Eq. (38) is identical to Eq. (35).

3. In case of classical plate theory in which shear deformation is not accounted for, Eq. (33) is specialized to give

$$
\Delta T_{c r 0}=\frac{\left(\bar{E}_{1} \bar{E}_{3}-\bar{E}_{2}^{2}\right) \pi^{2}}{\bar{E}_{1} P B_{h}^{2}(1+\nu)}\left[B_{a}^{2}+1+\left(\bar{T}_{11}^{0}\right)^{1 / 3}\right]
$$

where

$$
\bar{I}_{m n}^{0}=\frac{1024 \bar{E}_{1} m^{2} n^{2} \mu^{2} B_{a}^{4}}{9 \bar{A} \pi^{4} R_{m n}}
$$

4. Putting $k=0$, Eq. (33) is reduced to $\Delta T_{c r}$ for a isotropic homogeneous imperfect plate.

\subsection{Nonlinear temperature change across the thickness}

In this case, the temperature distribution through thickness is governed by the onedimensional Fourier equation of steady-state heat conduction as

$$
\frac{d}{d z}\left[K(z) \frac{d T}{d z}\right]=0, T(z=h / 2)=T_{c}, T(z=-h / 2)=T_{m}
$$

where $T_{c}, T_{m}$ are temperatures at ceramic-rich and metal-rich surfaces, respectively. The solution of Eq. (42) is obtained by means of polynomial series. Taking the first seven terms 
of series, solution for temperature distribution across the plate thickness becomes $[3,6,18]$

$$
T(z)=T_{m}+\Delta T \frac{r \sum_{p=0}^{6} \frac{\left(-r^{k} K_{c m} / K_{m}\right)^{p}}{p k+1}}{\sum_{p=0}^{6} \frac{\left(-K_{c m} / K_{m}\right)^{p}}{p k+1}}
$$

where $r=(2 z+h) / 2 h$ and $\Delta T=T_{c}-T_{m}$ is defined as the temperature difference between ceramic-rich and metal-rich surfaces of the plate.

By following the same procedure as the preceding loading case, and assuming the metal surface temperature as reference temperature, the critical buckling temperature difference is obtained for $m=n=1$ as

$$
\Delta T_{c r}=\frac{(1-\nu) \pi^{2}}{H B_{h}^{2}} \frac{\bar{F}\left(\frac{4 \bar{B}}{3 \bar{D}}+1\right) B_{h}^{2} R_{11}-\frac{16}{9}\left(\frac{\overline{E B}}{\bar{D}}-\bar{C}\right) \pi^{2} R_{11}^{2}+\pi^{2}\left(\bar{I}_{11}\right)^{1 / 3}}{\frac{\bar{F}}{\bar{D}} B_{h}^{2}+\left(1-\frac{4 \bar{E}}{3 \bar{D}}\right) \pi^{2} R_{11}}
$$

where

$$
H=\frac{\sum_{p=0}^{6} \frac{\left(-K_{c m} / K_{m}\right)^{p}}{p k+1}\left[\frac{E_{m} \alpha_{m}}{p k+2}+\frac{E_{m} \alpha_{c m}+E_{c m} \alpha_{m}}{(p+1) k+2}+\frac{E_{c m} \alpha_{c m}}{(p+2) k+2}\right]}{\sum_{p=0}^{6} \frac{\left(-K_{c m} / K_{m}\right)^{p}}{p k+1}} .
$$

Remarks: Similar to case of uniform temperature rise, when only the first order deformation is considered and when the shear deformation is ignored, Eq. (44) is reduced to results presented by Shariat and Eslami in works [19] and [18], respectively. Also, by setting $\mu=0$ or $k=0$, Eq. (44) is reduced to $\Delta T_{c r}$ for a perfect FGM plate or an isotropic homogeneous thick imperfect plate, respectively, under nonlinear temperature gradient. In general, by specializing Eq. (44) for thin and moderately thick plates with and without imperfection, we obtain limiting expressions of critical buckling temperature change being similar to Eqs. (34)-(41) in the previous loading case where $P$ is replaced by $H$. The detail procedure of obtaining these limiting expressions is omitted for brevity.

\section{RESULTS AND DISCUSSIONS}

The thermal buckling loads of the rectangular imperfect FGM plate are obtained in closed-form solutions for the assumed thermal loadings and are presented by Eqs. (33) and (44). These equations indicate that the critical buckling temperature change of an imperfect FGM plate is increased in comparison with a perfect one. The increase in $\Delta T_{c r}$ is expressed by an imperfection term $\left(\bar{I}_{11}\right)^{1 / 3}$, which directly depends on the imperfection size $\mu$. Also, investigation of Eq. (30) shows that the imperfection term is affected by the material and geometrical properties of a FGM plate. The fact that the thermal buckling load of a plate is increased by existence of geometrical imperfections is fully explained by Murphy and Ferreira [16] for isotropic homogeneous plates, extended by Shariat and Eslami $[18,19]$ for thin and moderately thick FGM plates. The present study again confirms this behavior for the thick FGM plates.

To illustrate the proposed approach, a rectangular imperfect functionally graded plate, that composed of ceramic and metal materials, is considered. The combination of materials consist of alumina (ceramic) and aluminum (metal) of which properties as follows $[3,6,18,19]$

$$
\begin{aligned}
E_{c} & =380 \mathrm{GPa}, \alpha_{c}=7.4 \times 10^{-6} /{ }^{o} \mathrm{C}, K_{c}=10.4 \mathrm{~W} / \mathrm{mK} . \\
E_{m} & =70 \mathrm{GPa}, \alpha_{m}=23.10^{-6} /{ }^{\circ} \mathrm{C}, K_{m}=204 \mathrm{~W} / \mathrm{mK}, \nu=0,3 .
\end{aligned}
$$


The plate is assumed to be simply supported on all edges.

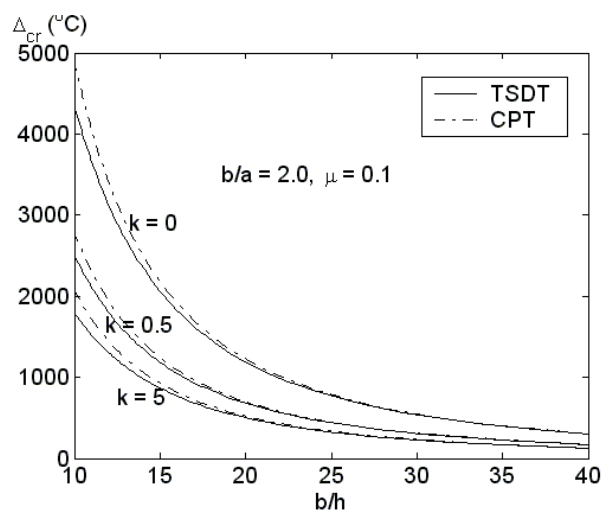

Fig. 1. $\Delta T_{c r}$ of FGM plate under uniform temperature rise vs. $b / h$

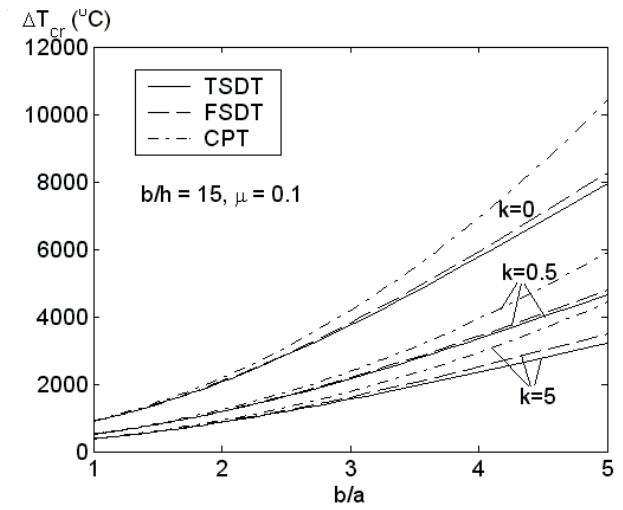

Fig. 3. $\Delta T_{c r}$ of FGM plate under uniform temperature rise vs. $b / a$

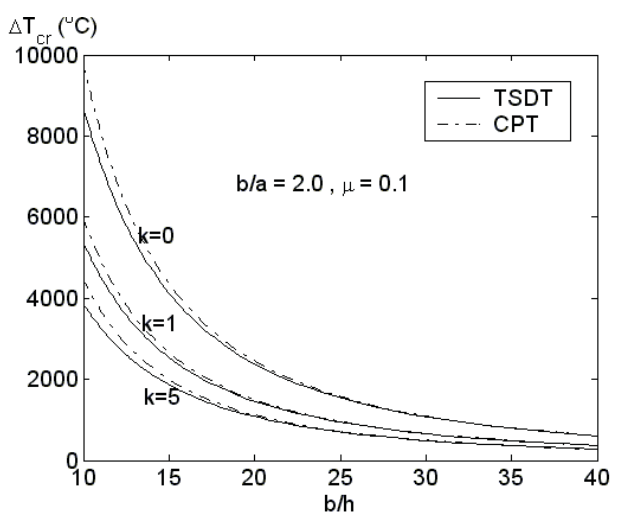

Fig. 2. $\Delta T_{c r}$ of FGM plate under nonlinear temperature change vs. $b / h$

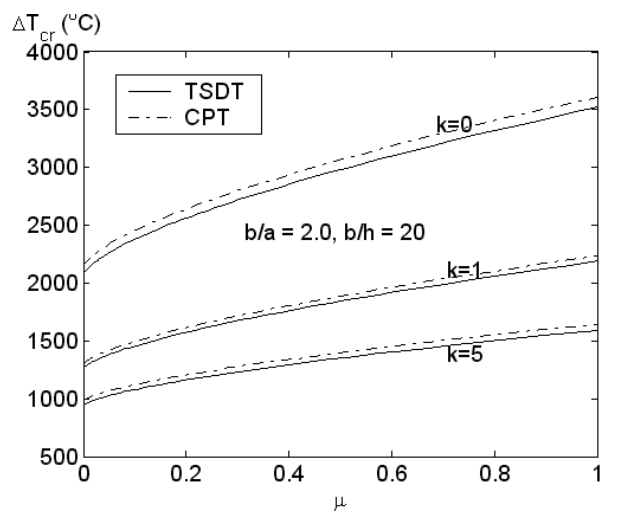

Fig. 4. $\Delta T_{c r}$ of FGM plate under nonlinear temperature change vs. $\mu$

The variation of the critical buckling temperature difference $\Delta T_{c r}$ versus side-tothickness ratio $b / h$ for FGM plates under two types of thermal loading are plotted in Figs. 1 and 2 for three various values of volume fraction index $k$. In these Figs., critical values are calculated by the third order shear deformation theory (TSDT) and are compared with the classical plate theory (CPT). As can be seen, $\Delta T_{c r}$ is reduced by increasing $b / h$ and $k$ as expected. Also, the CPT overestimates $\Delta T_{c r}$ than the TSDT, especially for thicker plates.

Fig. 3 shows variation trend of the $\Delta T_{c r}$ versus aspect ratio $b / a$ for the FGM plates under uniform temperature rise and by three various theories, the CPT, the FSDT, and the TSDT. As can be observed, $\Delta T_{c r}$ is considerably overpredicted when the shear deformation is not taken into consideration. In addition, discrepancy between predictions of $\Delta T_{c r}$ is increased when $b / a$ ratio to be large.

Variation of $\Delta T_{c r}$ for FGM plate under nonlinear temperature gradient versus imperfection size $\mu$ is illustrated in Fig. 4. Obviously, $\Delta T_{c r}$ is monotonically increased with increasing values of $\mu$ as mentioned. 


\section{CONCLUSION}

The present study extends foregoing works about thermal buckling of thin and moderately thick imperfect FGM plates to investigate buckling of thick imperfect FGM plates under thermal loadings. By analytical approach, one-term approximate solutions are chosen to satisfy governing equations and to derive closed-form relations of critical buckling temperature difference $\Delta T_{c r}$ for a simply supported imperfect FGM plate. The results show that $\Delta T_{c r}$ of an imperfect FGM plate is increased in comparison with that of a perfect plate under thermal loads. The results also confirm that the $\Delta T_{c r}$ of FGM plates is greatly effected by the geometry $(b / a$ and $b / h)$ and material $(k)$ parameters. Finally, it is recommended from the results that the higher order shear deformation theory should be used to analyze stability of thick plates and plates with large $b / a$ ratio for safe and reliable design.

\section{ACKNOWLEDGMENT}

This paper was supported by scientific research project of Vietnam National UniversityVNU, coded QGTD.09.01. The authors gratefully acknowledges this financial support.

\section{REFERENCES}

[1] D. O. Brush, B. O. Almroth, Buckling of Bars, Plates and Shells, McGraw-Hill, New York, 1975.

[2] R. Javaheri, M. R. Eslami, Buckling of functionally graded plates under in-plane compressive loading, ZAMM J 82 (4) (2002) 277-83.

[3] R. Javaheri, M. R. Eslami, Thermal buckling of functionally graded plates, AIAA J 40 (1) (2002) 162-9.

[4] R. Javaheri, M. R. Eslami, Thermal buckling of functionally graded plates based on higher order theory, J Thermal Stress 25 (1) (2002) 603-25.

[5] B.A. Samsam Shariat, M.R. Eslami, Buckling of thick functionally graded plates under mechanical and thermal loads, Compos Struct 78 (2007) 433-9.

[6] W. Lanhe, Thermal buckling of a simply supported moderately thick rectangular FGM plate, Compos Struct 64 (2004) 211-8.

[7] K-S. Na, J-H. Kim, Three-dimensional thermomechanical buckling analysis for functionally graded composite plates, Compos Struct 73 (2006) 413-22.

[8] J. N. Reddy, C. D. Chin, Thermomechanical analysis of functionally graded cylinders and plates, J Thermal Stress 21 (1998) 593-626.

[9] R. Shasiah, M. R. Eslami, Thermal buckling of functionally graded cylindrical shell, $J$ Thermal Stress 26 (3) (2003) 277-94.

[10] R. Shasiah, M. R. Eslami, Functionally graded cylindrical shell thermal instability based on improved Donnell equations, AIAA J 41 (9) (2003) 1819-24.

[11] W. Lanhe, Z. Jiang, Jun Liu, Thermoelastic stability of functionally graded cylindrical shells, Compos Struct $\mathbf{7 0}$ (2005) 60-8.

[12] M.R. Eslami, M. Shariyat, Elastic, plastic, and creep buckling of imperfect cylinders under mechanical and thermal loading, J. Pressure Vessel Tech. ASME 119 (1) (1997) 27-36.

[13] A. Mossavarali, Gh. Peydaye Saheli, M. R. Eslami, Thermoelastic buckling of isotropic and orthotropic plates with imperfections, J Thermal Stress 23 (2000) 853-72. 
[14] A. Mossavarali, M. R. Eslami, Thermoelastic buckling of plates with imperfections based on higher order displacement field, J Thermal Stress 25 (8) (2002) 745-71.

[15] M. R. Eslami, R. Shahsiah, Thermal buckling of imperfect cylindrical shells, J Thermal Stress 24 (2001) 71-89.

[16] K. D. Murphy, D. Ferreira, Thermal buckling of rectangular plates, Int. J. Solids Struct. 38 (2001) 3979-94.

[17] B.A. Samsam Shariat, R. Javaheri, M.R. Eslami, Buckling of imperfect functionally graded plates under in-plane compressive loading, Thin-wall Struct 43 (2005) 1020-36.

[18] B.A. Samsam Shariat, M.R. Eslami, Thermal buckling of imperfect functionally graded plates, Int. J. Solids Struct. 43 (2006) 4082-96.

[19] B.A. Samsam Shariat, M.R. Eslami, Effect of initial imperfection on thermal buckling of functionally graded plates, J Thermal Stress, 28 (2005) 1183-98.

[20] J. Yang, K. M. Liew, S. Kitipornchai, Imperfection sensitivity of thermal post-buckling behavior of higher-order shear deformable functionally graded plates, Int. J.Solids Struct. 43 (2006) 5247-66.

[21] J. N. Reddy, A.A. Khdeir, Buckling and vibration of laminated composite plates using various plate theories, AIAA J 27 (12) (1988) 1808-17.

Received August 20, 2009

\section{ỔN ĐỊNH NHIỆT ĐÀN HỒI CỦA TẤM DÀY BIẾN ĐỔI CHỨC NĂNG BAN ĐẦU KHÔNG PHẲNG}

Bài toán này nghiên cứu ổn định của các tấm dày làm từ vật liệu biến đổi chức năng khi chịu tải nhiệt và kể đến ảnh hưởng của imperfect hình dáng ban đầu. Các phương trình cân bằng, ổn định và tương thích của tấm dày biến đổi chức năng với imperfect được dẫn dắt bởi lý thuyết biến dạng trượt bậc ba của Reddy. Các tính chất vật liệu được giả thiết độc lập với nhiệt độ và biến đổi theo hướng chiều dày theo một hàm mũ. Các phương trình nhận được sẽ được giải theo phương pháp Galerkin để thu được các biểu thức hiển của nhiệt độ tới hạn. Sự phân tích ổn định cho một tấm chữ nhật imperfect, tựa bản trên các cạnh dưới tác dụng của hai loại tải nhiệt sẽ chỉ ra những ảnh hưởng của những tham số hình học và vật liệu, biến dạng trượt và imperfect lên nhiệt độ tới hạn của tấm

Appendix. Detail of coefficients $E_{i}$

$$
\begin{aligned}
E_{1} & =E_{m} h+\frac{E_{c m} h}{k+1}, E_{2}=\frac{E_{c m} h^{2} k}{2(k+1)(k+2)}, E_{3}=\frac{E_{m} h^{3}}{12}+E_{c m} h^{3}\left[\frac{1}{4(k+1)}-\frac{1}{(k+2)(k+3)}\right] \\
E_{4} & =\frac{E_{c m} h^{4}}{k+1}\left[\frac{1}{8}-\frac{3}{4(k+2)}+\frac{3}{(k+3)(k+4)}\right] \\
E_{5} & =\frac{E_{m} h^{5}}{80}+\frac{E_{c m} h^{5}}{k+1}\left[\frac{1}{16}-\frac{1}{2(k+2)}+\frac{3}{(k+2)(k+3)}-\frac{12}{(k+2)(k+4)(k+5)}\right] \\
E_{7} & =\frac{E_{m} h^{7}}{448}+\frac{E_{c m} h^{7}}{k+1}\left[\frac{1}{64}-\frac{6}{32(k+2)}+\frac{30}{16(k+2)(k+3)}-\frac{15}{(k+2)(k+3)(k+4)}\right. \\
& \left.+\frac{90}{(k+2)(k+3)(k+4)(k+5)}-\frac{360}{(k+2)(k+3)(k+4)(k+6)(k+7)}\right] .
\end{aligned}
$$

